\title{
A New Analytical Approach for Solving Van der Pol Oscillator
}

\author{
Md. Abul Kashem Mondal, Md. Helal Uddin Molla*, Md. Shamsul Alam \\ Department of Mathematics, Rajshahi University of Engineering and Technology (RUET), Rajshahi, Bangladesh \\ Email address: \\ info.abulkashem@gmail.com (Md. A. K. Mondal), helal.mathruet@gmail.com (Md. H. U. Molla), msalam1964@yahoo.com (Md. S. Alam) \\ ${ }^{*}$ Corresponding author
}

\section{To cite this article:}

Md. Abul Kashem Mondal, Md. Helal Uddin Molla, Md. Shamsul Alam. A New Analytical Approach for Solving Van der Pol Oscillator. Science Journal of Applied Mathematics and Statistics. Vol. 7, No. 4, 2019, pp. 51-55. doi: 10.11648/j.sjams.20190704.11

Received: July 20, 2019; Accepted: September 16, 2019; Published: October 9, 2019

\begin{abstract}
The Van der Pol oscillator is a nonlinear damping and non-conservative oscillator. Energy is generated at low amplitude and dissipated at high amplitude. This nonlinear oscillator was first introduced by Dutch electrical engineer and physicist B. Van der Pol and it was originally used to investigate vacuum tubes. Nowadays, it is used in both physical and biological sciences. It is also used in sociology and even in economics. It has a limit cycle and in earlier it was determined by the classical perturbation methods when the nonlinear term is small. Then the harmonic balance method was used to determine the limit cycle for stronger nonlinear case. Moreover, many researchers have been analyzed this oscillator by various numerical approaches. In this article, a new analytical approach based on harmonic balance method is presented to determine the limit cycle as well as approximate solutions of this nonlinear oscillator. The frequency as well as the limit cycle obtained by new approach has been compared with those obtained by other existing methods. The present method gives better result than other existing results and also close to the corresponding numerical result (considered to the exact result). Moreover, the present method is simpler than the existing harmonic balance method.
\end{abstract}

Keywords: Van der Pol Oscillator, Limit Cycle, Harmonic Balance Method, Frequency

\section{Introduction}

Van der Pol oscillation has become the interest of many researchers because of its various applications in human activities, sciences, technologies and industrial applications. Thus, in present time, nonlinear processes are one of the biggest challenges and not easy to control because the nonlinear characteristic of the system abruptly changes due to some small changes of valid parameters including time. The general theory and solution technique for linear differential equations in both science and engineering as well as in other disciplines have extensively developed. Yet very often many oscillatory systems are governed by nonlinear differential equation. Although it is possible in many cases to replace the nonlinear differential equation by a corresponding linear differential equation which approximates the original equation, such linearization is not always feasible or possible. In such cases, the actual differential equations must be directly dealt with. Many researchers have been determined the approximate solutions of nonlinear problems by using various methods such as perturbation method which is originally developed for handling weak nonlinear problems in which small parameters exist [1-3]. The parameters are analytically expanded into power series of the parameter. The coefficients of the series are found as solutions of a set of linear algebraic problem. However, in both science and engineering, there exist many nonlinear problems without small parameters. Even if there exists such a parameter, the analytical solution determined by the perturbation method has a small validity. Thus, many approximation methods were developed for solving strongly nonlinear oscillators, including modified Lindstedt-Poincare method [4-5], harmonic balance method [6-10], residue harmonic balance method [11], global residue harmonic balance method [12], iterative homotopy harmonic balance method [13], amplitude-frequency formulation [14] and homotopy analysis method [15]. Moreover energy balance method is used technique for solving strongly nonlinear oscillators [16-21]. A detailed review on some recently developed nonlinear analytical methods can be found in [22-28]. Also in a recent work, Rahman et al. has used harmonic balance method to determine an approximate 
solution of Van der Pol oscillator [10]. But the method is not a simple one. However, the approximate solutions obtained by these methods are not significantly improved. On the other hand, any particular method is not suitable for all problems i.e., the special method is appropriate for the special nonlinear problems. Also, the solution procedure of some methods is laborious and difficult as well as results are not so closed to exact results. Also, these methods involve tedious derivations and computations, and they are difficult to implement. In an attempt to improve the accuracy of the existing analytical methods, a new analytical approach based on harmonic balance method has been presented to obtain the approximate solution of Van der Pol nonlinear oscillator.
The obtained results have been compared with those obtained by Rahman et al. [10].

\section{Formulation and Solution Method}

Let us consider a nonlinear differential equation

$$
\ddot{x}+x=\varepsilon f(x, \dot{x}), \quad x(0)=a, \dot{x}(0)=0,
$$

where, over dot denotes the derivative with respect to time $t$, $f(x, \dot{x})$ is a nonlinear function and $\varepsilon$ is constant. Consider a periodic solution as in the form:

$$
\begin{aligned}
x(t) & =2 \cos \varphi+\delta \cos \varphi+p(\sin 3 \varphi-3 \sin \varphi)+q(\sin 5 \varphi-5 \sin \varphi) \varphi) \\
& +u(\cos 3 \varphi-\cos +v(\cos 5 \varphi-\cos \varphi)+w(\cos 7 \varphi-\cos \varphi)+\ldots)
\end{aligned}
$$

where $0<\delta<<1, p, q, u, v, w$ are constants, $\phi=\omega t$ and $\omega=2 \pi / T$ is a frequency of nonlinear oscillation, here $T$ is a period. Solution Eq.(2) readily satisfies the initial conditions $x(0)=a, \dot{x}(0)=0$.

Substituting Eq. (2) in Eq. (1) and expanding $f(x, \dot{x})$ in a Fourier series, it turns to an algebraic identity

$$
\begin{aligned}
& (-2+u+v+w-\delta)\left(\omega^{2}-1\right) \cos \phi+u\left(1-9 \omega^{2}\right) \cos 3 \phi+\cdots \\
& +(3 p+5 q)\left(\omega^{2}-1\right) \sin \phi+p\left(1-9 \omega^{2}\right) \sin 3 \phi+\cdots \\
& =\varepsilon\left[F_{1}(p, q, \omega, u, v, w, \delta) \cos \phi+F_{3}(p, q, \omega, u, v, w, \delta) \cos 3 \phi+\cdots\right. \\
& \left.+G_{1}(p, q, \omega, u, v, w, \delta) \sin \phi+G_{3}(p, q, \omega, u, v, w, \delta) \sin 3 \phi+\cdots \cdots\right]
\end{aligned}
$$

Equating the coefficients of equal harmonics of Eq. (3), we obtain the algebraic equations are

$$
\begin{gathered}
(-2+u+v+w-\delta)\left(\omega^{2}-1\right)=\varepsilon F_{1}, u\left(1-9 \omega^{2}\right)=\varepsilon F_{3}, \\
v\left(1-25 \omega^{2}\right)=\varepsilon F_{5}, \cdots \\
(3 p+5 q)\left(\omega^{2}-1\right)=\varepsilon G_{1}, p\left(1-9 \omega^{2}\right)=\varepsilon G_{3}, \\
q\left(1-25 \omega^{2}\right)=\varepsilon G_{5}, \cdots .
\end{gathered}
$$

Since $\delta$ is much smaller than 1 so all the constants $\omega, u, v, w, p, q$, of Eqs.(4) can be expanded inpowers of $\delta$ as

$$
\begin{aligned}
& \omega=1+c_{1} \delta+d_{1} \delta^{2}+\cdots \\
& u=c_{2} \delta+d_{2} \delta^{2}+\cdots \\
& v=c_{3} \delta+d_{3} \delta^{2}+\cdots \\
& w=c_{4} \delta+d_{4} \delta^{2}+\cdots \\
& p=c_{5} \delta+d_{5} \delta^{2}+\cdots \\
& q=c_{6} \delta+d_{6} \delta^{2}+\cdots
\end{aligned}
$$

Now Eqs. (4) can be solved for $c_{1}, c_{2}, c_{3}, c_{4}, c_{5}, c_{6}, d_{1}, d_{2}, d_{3}, d_{4}, d_{5}, d_{6}$ and $\delta$. Substituting the values of $\omega, u, v, w, p, q$ and $\delta$ from Eqs. (5) into Eq. (1) we obtain the solutions.

\section{Example}

To illustrate the accuracy of the proposed method one example has been presented in this section. Consider the Van der Pol nonlinear conservative oscillator as in the following form

$$
\ddot{x}+x=\varepsilon\left(1-x^{2}\right) \dot{x}, \quad x(0)=a, \dot{x}(0)=0 .
$$

Consider a periodic solution as in the form:

$$
\begin{aligned}
x(t) & =2 \cos \varphi+\delta \cos \varphi+p(\sin 3 \varphi-3 \sin \varphi)+q(\sin 5 \varphi-5 \sin \varphi) \varphi) \\
& +u(\cos 3 \varphi-\cos +v(\cos 5 \varphi-\cos \varphi)+w(\cos 7 \varphi-\cos \varphi)+\ldots)
\end{aligned}
$$

Substituting Eq. (7) into Eq. (6) and expanding in a Fourier series and equating the coefficients of $\cos \phi$, $\cos 3 \phi$, $\cos 5 \phi, \cos 7 \phi$ and $\sin \phi, \sin 3 \phi, \sin 5 \phi$ respectively, we obtained the following equations as

$$
\begin{aligned}
& \left(8-4 w+4 \delta+\varepsilon \omega\left(4 p-42 p^{3}-168 p^{2} q-246 p q^{2}-135 q^{3}-14(p+2 q) u^{2}-3(2 p+5 q) v^{2}\right.\right. \\
& \left.+8 p w+16 q w-8 p w^{2}-13 q w^{2}-8 p \delta-20 q \delta+4 p w \delta+8 q w \delta-2 p \delta^{2}-5 q \delta^{2}\right)-8 \omega^{2} \\
& +4 w \omega^{2}-4 \delta \omega^{2}-2 u\left(2+q(6 v+9 w-11(2+\delta)) \varepsilon \omega+p(6 w-5(2+\delta)) \varepsilon \omega-2 \omega^{2}\right) \\
& \left.+2 v\left(p(2+3 w+\delta) \varepsilon \omega+q(10+w+5 \delta) \varepsilon \omega+2\left(\omega^{2}-1\right)\right)\right) / 4=0 .
\end{aligned}
$$




$$
\begin{aligned}
& \varepsilon \omega\left(-18 q u^{2} \varepsilon \omega+\left(84 p^{3}+270 p^{2} q+3 p\left(-8+87 q^{2}+12 v-3 v^{2}-10 v w+3 w^{2}-4 \delta\right.\right.\right. \\
& \left.\left.+6 v \delta-\delta^{2}\right)+2 q\left(25 q^{2}-3\left(7 v^{2}+w^{2}-3 w(2+\delta)+2(2+\delta)^{2}+3 v(4 w-3(2+\delta))\right)\right)\right) / 4 \\
& \left.+u\left(4+6 p(2-6 v+3 w+\delta) \varepsilon \omega+6 q(10-15 v+w+5 \delta) \varepsilon \omega-36 \omega^{2}\right)\right)=0 . \\
& 5(p+3 q) v^{2} \varepsilon \omega-5\left(12 p^{3}+15 p^{2} q-p\left(4+35 q^{2}+8 u^{2}+4 w-3 w^{2}+4 \delta+2 w \delta+\delta^{2}\right.\right. \\
& -2 u(6+2 w+3 \delta))-q\left(51 q^{2}+19 u^{2}-2 u(4 w+7(2+\delta))+2\left(2-3 w^{2}+4 \delta+\delta^{2}\right.\right. \\
& +3 w(2+\delta)))) \varepsilon \omega+2 v\left(2-5 q(4-7 u+3 w+2 \delta) \varepsilon \omega-5 p(2-3 u+\delta) \varepsilon \omega-50 \omega^{2}\right)=0 \text {. } \\
& 7 q w^{2} \varepsilon \omega+7\left(3 p^{3}-10 p^{2} q+q\left(4-25 q^{2}-6 u^{2}-24 v+22 u v+11 v^{2}+4 \delta-12 v \delta+\delta^{2}\right)\right. \\
& \left.-p\left(39 q^{2}+5 u^{2}+v(8-3 v+4 \delta)-2 u(2+4 v+\delta)\right)\right) \varepsilon \omega+2 w(2-7 p(u-2 v) \varepsilon \omega \\
& \left.-7 q(2-6 v+\delta) \varepsilon \omega-98 \omega^{2}\right)=0 \text {. } \\
& -q^{2} \varepsilon \omega(54-62 u-27 v-17 w+27 \delta)+p^{2}\left(14 u+6 w-5(2+\delta) \varepsilon \omega+\left(2 u^{3}+3 v^{3}+8 w\right.\right. \\
& -10 w^{2}+3 w^{3}-8 \delta+12 w \delta-5 w^{2} \delta-6 \delta^{2}+3 w \delta^{2}-\delta^{3}+v^{2}(7 w-5(2+\delta))+u^{2}(4 v \\
& +2 w-3(2+\delta))+v\left(8+7 w^{2}+12 \delta+3 \delta^{2}-8 w(2+\delta)\right)+2 u\left(2+3 v^{2}+2 w^{2}-3 v(2\right. \\
& \left.\left.-w+\delta)+4 \delta+\delta^{2}-2 w(2+\delta)\right)\right) \varepsilon \omega-20 q\left(1-\omega^{2}\right)+2 p(q(-22+28 u+6 v \\
& \left.+9 w-11 \delta) \varepsilon \omega-6\left(1-\omega^{2}\right)\right)=0 \text {. } \\
& -3 p^{2}(28 u-3(6+2 v-5 w+3 \delta)) \varepsilon \omega-\left(8+8 u^{3}-6 v^{2}+2 v^{3}-12 w+12 v w+6 w^{2}\right. \\
& -6 v w^{2}-w^{3}-3 q^{2}(70-97 u-10 v-24 w+35 \delta)+12 \delta-3 v^{2} \delta-12 w \delta+6 v w \delta \\
& +3 w^{2} \delta+6 \delta^{2}-3 w \delta^{2}+\delta^{3}+3 u^{2}(2 v+w-3(2+\delta))+3 u\left(3 v^{2}+w^{2}+\delta(4+\delta)\right. \\
& -2 v(2+w+\delta))) \varepsilon \omega+2 p\left(2+3 q(34-50 u+3 v-19 w+17 \delta) \varepsilon \omega-18 \omega^{2}\right)=0 \text {. } \\
& 5 q^{2}(25 u-51 v+25 w) \varepsilon \omega+5\left(-4 v+8 v^{2}-3 v^{3}-4 w+12 v w-5 v^{2} w+4 w^{2}-6 v w^{2}\right. \\
& -w^{3}+u^{2}(2+5 v+\delta)-5 p^{2}(2-4 u+3 v-4 w+\delta)-8 v \delta+4 v^{2} \delta-4 w \delta+6 v w \delta \\
& \left.+2 w^{2} \delta-2 v \delta^{2}-w \delta^{2}-u\left(5 v^{2}-2 v(6-4 w+3 \delta)+(2-w+\delta)^{2}\right)\right) \varepsilon \omega+2 q(2 \\
& \left.-5 p(10-25 u+25 v-24 w+5 \delta) \varepsilon \omega-50 \omega^{2}\right)=0 \text {. }
\end{aligned}
$$

Since $\delta$ is much smaller than 1 so all the constants $\omega, u, v, w, p, q$ of Eqs.(8)-(14) can be expanded in powers of $\delta$ as in the following form:

$$
\begin{aligned}
& \omega=1+c_{1} \delta+d_{1} \delta^{2}+\cdots \\
& u=c_{2} \delta+d_{2} \delta^{2}+\cdots \\
& v=c_{3} \delta+d_{3} \delta^{2}+\cdots \\
& w=c_{4} \delta+d_{4} \delta^{2}+\cdots \\
& p=c_{5} \delta+d_{5} \delta^{2}+\cdots \\
& q=c_{6} \delta+d_{6} \delta^{2}+\cdots
\end{aligned}
$$

Substituting (15) into (8)-(12) and (14) and equating the coefficients of $\delta$ we obtain a system of linear equations of $c_{1}, c_{2}, c_{3}, \cdots, d_{1}, d_{2}, d_{3}, \cdots$. Solving these equations and substituting the values of $c_{1}, c_{2}, c_{3}, \cdots, d_{1}, d_{2}, d_{3}, \cdots$ into (9) we obtain for $\varepsilon=1$ :

$$
\omega=1-\frac{1754 \delta}{1581}-\frac{2958662347157 \delta^{2}}{71132506938}+\cdots
$$

$$
\begin{gathered}
u=\frac{2274 \delta}{527}+\frac{750917710871 \delta^{2}}{7903611882}+\cdots \\
v=-\frac{5035 \delta}{4743}-\frac{2676227662505 \delta^{2}}{426795041628}+\cdots \\
w=-\frac{455 \delta}{4743}-\frac{4091164395829 \delta^{2}}{426795041628}+\cdots \\
p=-\frac{7016 \delta}{1581}-\frac{2082541407358 \delta^{2}}{35566253469}+\cdots \\
q=-\frac{1040 \delta}{1581}-\frac{1119969952960 \delta^{2}}{35566253469}+\cdots
\end{gathered}
$$

Substituting (16) into (13) we obtain another series. 


$$
-2+\frac{18146 \delta}{527}+\frac{44198163782443 \delta^{2}}{47421671292}+\frac{2295665100755905642 \delta^{3}}{168690740203467}+\cdots=0
$$

From this series (17) we obtain the value of $\delta$ and use the smallest $|\delta|$ in Eq.(16). Finally, substituting the value of all constants in Eq. (7) we obtain the required solution.

\section{Results and Discussion}

To test the accuracy of an approximate solution, some authors compared analytical solutions to those obtained by the numerical techniques $[9,27]$. Here, we have been presented a new analytical approach based on harmonic balance method to determine the approximate solution of Van der Pol nonlinear equation. The solutions have been compared with numerical solution for $\varepsilon=1$ which are presented in figure 1. Also the solution of Rahman et al [10] for Van der Pol oscillator together with numerical solution for $\varepsilon=1$ has been provided in figure 2. The corresponding numerical results have been calculated by Runge-Kutta fourth order formula. From the figures we observe that the present solutions are nicely agreement with the corresponding numerical solutions and gives almost similar results to those obtained by Rahman et al [10]. Though the present solution is almost similar to that of Rahman et al [10] but present method is much easier than that of Rahman et al [10]. Moreover in the article of Rahman et al. [10] two simultaneous algebraic-transcendental equations were solved which is laborious process. But in our present method a cubical equation has been solved (Eq. (17)).

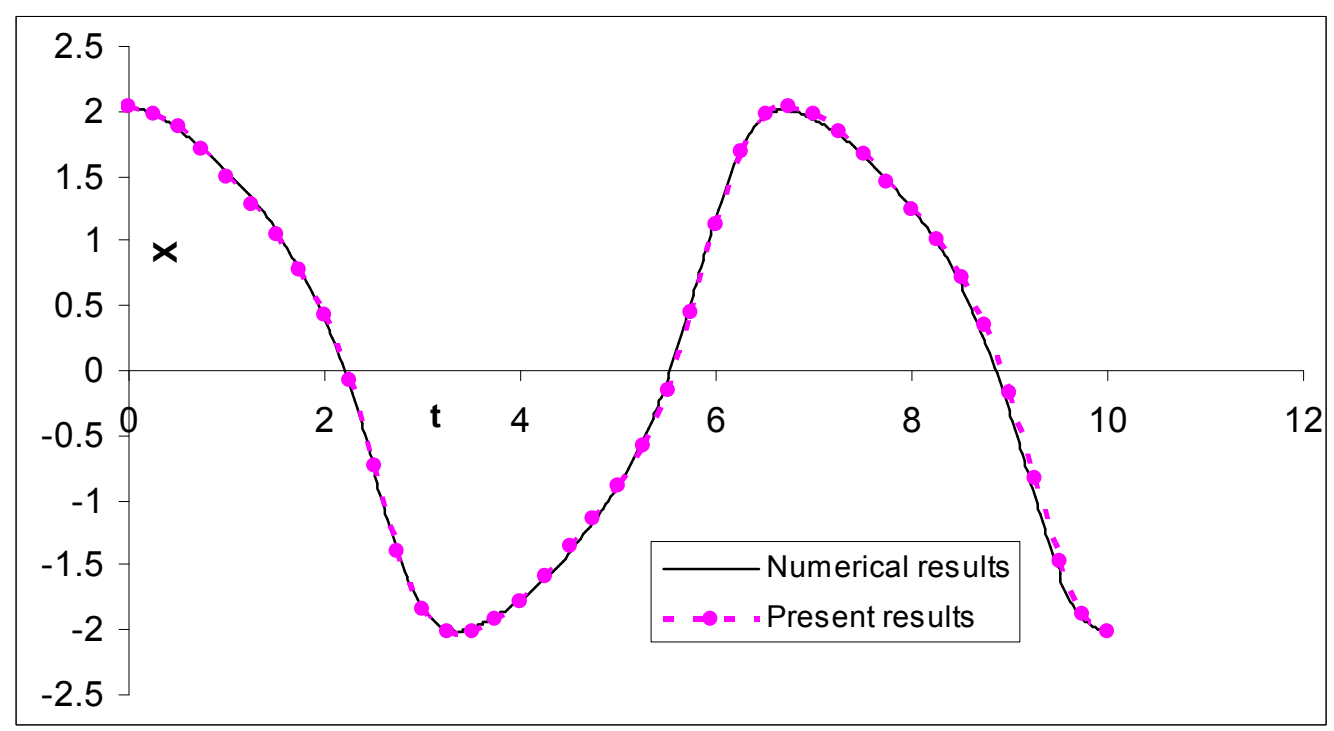

Figure 1. Comparison of present results with numerical resultsfor $\varepsilon=1$.

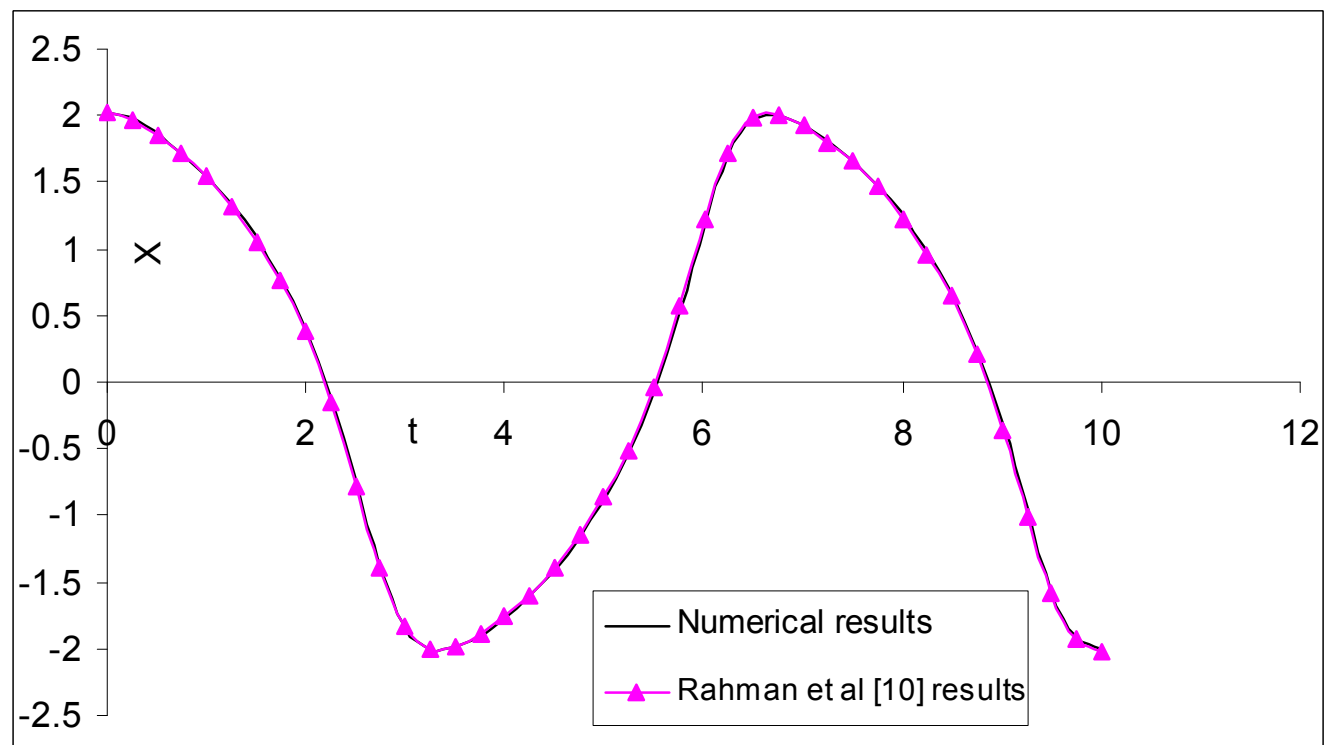

Figure 2. Comparison of Rahman et al [10] results with numerical results for $\varepsilon=1$. 


\section{Conclusion}

An analytical technique based on the harmonic balance method has been presented to obtain approximate solution of Van der Pol oscillator as well as its limit cycle when the coefficient of the nonlinear term, $\varepsilon$ is not small. Earlier perturbation method was used to investigate this oscillator when $\varepsilon$ is small. In a recent article [10] harmonic balance method has been used to solve this equation when $\varepsilon=1$. But the method is more complicated than the present technique. A new parameter has been introduced for solving the nonlinear algebraic equations related to the coefficients of higher harmonic terms and the frequency of oscillation. The solution has been compared with numerical and other existing solution. Comparing the results we say that the present method shows a good coincidence with the numerical method.

\section{References}

[1] Krylov NN, Bogoliubov NN (1947), Introduction to Nonlinear Mechanics. Princeton University Press, New Jersey.

[2] Nayfeh AH (1973), Perturbation Methods. John Wiley and Sons, New York.

[3] Kovacic I and Mickens RE (2012), A generalized van der Pol type oscillator: Investigation of the properties of its limit cycle. J. Math. Comput. Model. 55, 645-653.

[4] Cheung YK, Chen SH and Lau SL (1991), A modified Lindstedt-Poincare method for certain strongly non-linear oscillators. Int. J. Nonlin. Mech. 26 (3), 367-378.

[5] Liu HM (2005), Approximate period of nonlinear oscillators with discontinuities by modified Lindstedt-Poincare method. Chaos, Solit. Fract. 23, 577-579.

[6] Mickens RE (2001), A generalization of the method of harmonic balance. J. Sound Vib. 111, 515-518.

[7] Alam MS, Razzak MA, Hosen MA and Parvez MR (2016), The rapidly convergent solutions of strongly nonlinear oscillators. Spring. Plu. 5, 1258, 16 pages.

[8] Hosen MA and Chowdhury MSH (2015), A new analytical technique based on harmonic balance method to determine approximate periods for Duffing-harmonic oscillator. Alexandria Eng. J. 54, 233-239.

[9] Lim CW and Lai SK (2006), Accurate higher-order analytical approximate solutions to non-conservative nonlinear oscillators and application to Van der Pol damped oscillations. Int. J. Mech. Sci., 48, 483-492.

[10] Rahman MS, Haque ME and Shanta SS (2010), Harmonic balance solution of nonlinear differential equation (Nonconservative). J. Advan. Vib. Engin., 9 (4), 345-356.

[11] GuoZ and Ma X (2014), Residue harmonic balance solution procedure to nonlinear delay differential systems. J. Applied Math. Comp. 237, 20-30.

[12] $\mathrm{Ju} \mathrm{P}$ and Xue X (2015), Global residue harmonic balance method for large-amplitude oscillations of a nonlinear system. J. Applied Math. Model. 39, 449-454.

[13] Guo Z and Leung AYT (2010), The iterative homotopy harmonic balance method for conservative HelmholtzDuffing oscillators. J. Applied Math. Comp. 215, 3163-3169.

[14] El-Naggar AM and Ismail GM (2012), Applications of He's amplitude-frequency formulation to the free vibration of strongly nonlinear oscillators. J. Applied Math. Sci. 6, 20712079 .

[15] Mishara V, Das S, Jafari H and Ong SH (2016), Study of fractional order van der Pol equation. J. King Saud Univ. Sci. 28, 55-60.

[16] Khan Y and Mirzabeigy A (2014), Improved accuracy of He's energy balance method for analysis of conservative nonlinear oscillator. Neural Comput. Appli. 25, 889-895.

[17] Molla MHU, Razzak MA and Alam MS (2018), A more accurate solution of nonlinear conservative oscillator by energy balance method. Multidis. Model. Mater. Struct. 14 (4), 634-646.

[18] Mehdipour I, Ganji DD and Mozaffari M (2010), Application of the energy balance method to nonlinear vibrating equations. Current Appli. Phys. 10, 104-112.

[19] Babazadeh H, Ganji DD and Akbarzade M (2008), He's energy balance method to evaluate the effect of amplitude on the natural frequency in nonlinear vibration systems. Progress In Electromag. Res. M, 4, 143-154.

[20] Molla MHU, Razzak MA and Alam MS (2017), An analytical technique for solving quadratic nonlinear oscillator. Multidis. Model. Mater. Struct. 13 (3), 424-433.

[21] Molla MHU and Alam MS (2017), Higher accuracy analytical approximations to nonlinear oscillators with discontinuity by energy balance method. Result. Phys. 7, 2104-2110.

[22] Belendez A, Arribas E, Ortuno M, Gallego S, Márquez A and Pascual I (2012), Approximate solutions for the nonlinear pendulum equation using a rational harmonic representation. Comput. Math. Appli. 64, 1602-1611.

[23] Alam MS, Haque ME and Hossain MB (2007), A new analytic tecnique to find periodic solutions of nonlinear systems. Int. J. Nonlinear Mech. 42, 1035-1045.

[24] Liping Liu, Earl. H. Dowell, Kenneth C. Hall (2007), A novel harmonic balance analysis for the Van der Pol oscillator, Int. J. Non-Linear Mech. 42, 2-12.

[25] Shen Y, Yang S, Sui C (2014), Analysis on limit cycle of fractional-order van der Pol oscillator, Chaos, Solitons and Fractals, 67, 94-102.

[26] Zhang J, Gu X (2010), A Stability and bifurcation analysis in the delay-coupled van der Pol oscillators, Applie. Math. Model. 34, 2291-2299.

[27] Barro MA (2016), Stability of a ring of coupled van der Pol oscillators with non-uniform distribution of the coupling parameter, J. Appli. Res. Techno. 14, 62-66.

[28] Casaleiroa J, Oliveira LB, Pintoa AC (2014), Van der Pol Approximation Applied to Wien Oscillators, Procedia Techno. $17,335-342$. 